\title{
Assessment of COVID-19 progression on day 5 from symptoms onset
}

\author{
Elisa Gentilotti ${ }^{1}$, Alessia Savoldi ${ }^{1}$, Monica Compri ${ }^{1}$, Anna Górska ${ }^{1}$, Pasquale De Nardo ${ }^{1 *}$, Alessandro Visentin ${ }^{1}$, \\ Giorgia Be ${ }^{1}$, Elisa Razzaboni ${ }^{1}$, Nicola Soriolo' ${ }^{1}$ Dario Meneghin' ${ }^{1}$ Domenico Girelli², Claudio Micheletto ${ }^{3}$, \\ Sara Mehrabi ${ }^{4}$, Elda Righi ${ }^{1}$ and Evelina Tacconelli ${ }^{1}$
}

\begin{abstract}
Background: A major limitation of current predictive prognostic models in patients with COVID-19 is the heterogeneity of population in terms of disease stage and duration. This study aims at identifying a panel of clinical and laboratory parameters that at day- 5 of symptoms onset could predict disease progression in hospitalized patients with COVID-19.

Methods: Prospective cohort study on hospitalized adult patients with COVID-19. Patient-level epidemiological, clinical, and laboratory data were collected at fixed time-points: day 5, 10, and 15 from symptoms onset. COVID-19 progression was defined as in-hospital death and/or transfer to ICU and/or respiratory failure $\left(\mathrm{PaO}_{2} / \mathrm{FiO}_{2}\right.$ ratio $\left.<200\right)$ within day-11 of symptoms onset. Multivariate regression was performed to identify predictors of COVID-19 progression. A model assessed at day- 5 of symptoms onset including male sex, age $>65$ years, dyspnoea, cardiovascular disease, and at least three abnormal laboratory parameters among CRP (>80 U/L), ALT (>40 U/L), NLR (>4.5), LDH (> $250 \mathrm{U} / \mathrm{L})$, and $\mathrm{CK}(>80 \mathrm{U} / \mathrm{L})$ was proposed. Discrimination power was assessed by computing area under the receiver operating characteristic (AUC) values.
\end{abstract}

Results: A total of 235 patients with COVID-19 were prospectively included in a 3-month period. The majority of patients were male $(148,63 \%)$ and the mean age was 71 (SD 15.9). One hundred and ninety patients (81\%) suffered from at least one underlying illness, most frequently cardiovascular disease (47\%), neurological/psychiatric disorders (35\%), and diabetes (21\%). Among them 88 (37\%) experienced COVID-19 progression. The proposed model showed an AUC of 0.73 ( $95 \% \mathrm{Cl} 0.66-0.81)$ for predicting disease progression by day-11.

Conclusion: An easy-to-use panel of laboratory/clinical parameters computed at day-5 of symptoms onset predicts, with fair discrimination ability, COVID-19 progression. Assessment of these features at day-5 of symptoms onset could facilitate clinicians' decision making. The model can also play a role as a tool to increase homogeneity of population in clinical trials on COVID-19 treatment in hospitalized patients.

Keywords: COVID-19, Disease progression, Outcome, Public health

\footnotetext{
${ }^{*}$ Correspondence: pasquale.denardo@univr.it

1 Division of Infectious Diseases, Department of Diagnostics and Public

Health, University of Verona, P.le L.A. Scuro 10, 37134 Verona, Italy

Full list of author information is available at the end of the article
}

\section{Introduction}

The global Coronavirus disease 2019 (COVID-19) pandemic is challenging healthcare systems worldwide [1]. As of June 2021, the number of patients confirmed to have COVID-19 has exceeded 190 million in 191 countries, with more than 4 million deaths [2]. The critical disease, described in up to $20 \%$ of hospitalised patients 
$[3,4]$, is associated with high case fatality rate and leads to substantial increase in the demand for hospital beds and shortage of medical equipment. Up to January 2021, the hospital and intensive care unit (ICU) occupancy and the new admission due to COVID-19 increased in several European countries, reaching $82 \%$ of the peak ICU occupancy observed during the pandemic [5]. Descriptive studies on the natural course of COVID-19 have revealed that the disease progression occurs typically at an early stage of the illness, usually within 7-14 days after the onset of symptoms $[6,7]$ with or without acute severe respiratory distress syndrome [8]. Given the lack of standardized treatments for patients with COVID-19, the identification of patients at higher risk of short-term complications is of utmost importance to ensure the best possible clinical care and to optimise resource allocation. Since the beginning of the pandemic, several prognostic predictive models combining various clinical and laboratory parameters have been developed to estimate risk of patients experiencing poor outcomes. A systematic review of ten prognostic models observed that the most reported predictors of disease progression and mortality were age, sex, C-reactive protein (CRP), lactic dehydrogenase (LDH) and lymphocyte count [9].

All the published models were computed using the trend of clinical and laboratory information starting from the admission day onward [10-12], with the result that the data was related to a wide range of disease length as opposed to specific time points. To the best of our knowledge, no prognostic models have been developed taking into account a homogeneous time point across patients, such as the onset of symptoms, for the assessment of disease progression.

This study aims at identifying a panel of clinical and laboratory parameters that could support the prediction of disease progression based on symptoms onset in hospitalized patients with COVID-19.

\section{Methods}

\section{Study design and participants}

This is a prospective cohort study. All adult patients (aged $\geq 18$ years) with microbiologically confirmed SARS-CoV-2 infection admitted from 1st March to 31st May, 2020 at the University Hospital of Verona were prospectively followed during the hospital stay and included in the COVID 19-VR registry (registered on ClinicalTrials.gov, 18/05/2020, Number: NCT04497194). The study protocol is performed in accordance with the relevant guidelines. The study was approved by the hospital Institutional Review Board ("Comitato etico per la sperimentazione clinica delle province di Verona e Rovigo", 2577CESC). A written informed consent for study participation was obtained from the patients or from the legal guardian on enrolment. Infection with SARS-CoV-2 was microbiologically defined by a positive real-time reversetranscriptase polymerase chain reaction (RT-PCR) assay from nasopharyngeal swab.

\section{Data collection}

Patient-level demographic (age, sex, ethnicity), chronic underlying diseases (hypertension and/or coronary artery disease, diabetes, dyslipidaemia, pulmonary disease (chronic obstructive pulmonary disease, active tuberculosis, asthma or obstructive sleep apnoea syndrome), chronic kidney disease, neoplastic disease, thyroid disorders, neurological/psychiatric disorders, liver diseases, obesity (body mass index higher than 30), and clinical parameters (body temperature, respiratory rate, blood pressure, heart rate, peripheral oxygen saturation) were gathered at study inclusion and entered into a predefined case report form. Laboratory parameters, including lymphocytes (reference range: 120-400 10\%/L) and neutrophils (reference range: $180-80010^{9} / \mathrm{L}$ ) count, LDH (reference range: 135-214 U/L), CRP (reference range: $<5 \mathrm{mg} / \mathrm{L}$ ), aspartate aminotransferase (AST, reference range: 5-45 U/L), alanine aminotransferase (ALT, reference range: $5-45 \mathrm{U} / \mathrm{L})$ ), creatine kinase $(\mathrm{CK}$, reference range: $30-200 \mathrm{U} / \mathrm{L}$ ) were extracted from routine blood testing at fixed time points: at hospital admission, day-5 $( \pm 1)$ and day-10 $( \pm 1)$ from onset of symptoms and in case of transfer to ICU/sub-intensive unit, discharge, and death.

\section{Outcomes}

COVID-19 progression was defined as $\mathrm{PaO}_{2} / \mathrm{FiO}_{2}$ ratio $<200$ and/or transfer to ICU and/or in-hospital death occurring by day-11 after symptoms onset.

\section{Statistical analysis}

Descriptive statistics included means with standard deviations (SD) and frequency analyses (percentages) for categorical variables at day 5 after onset of symptoms. T-test for independent samples, $\chi^{2}$ test, or Fisher's test were applied to compare differences between patients with or without disease progression. Logistic regression was performed to evaluate univariate and multivariate associations with the outcome. Results were presented as odds ratio (OR) and $95 \%$ confidence interval $(95 \% \mathrm{CI})$. A $p$-value less than 0.05 was deemed as statistically significant. In order to enter the model as categorical variable, the cut-off of each laboratory parameter was set at the best point of sensitivity and specificity identified through the receiver operating characteristic (ROC) curve. The selected parameters with correspondent cut-offs were: $\mathrm{CRP}>80 \mathrm{mg} / \mathrm{L}$, neutrophil lymphocyte ratio $(\mathrm{NLR})>4.5, \mathrm{LDH}>250 \mathrm{U} / \mathrm{L}$, 
$\mathrm{CK}>80 \mathrm{U} / \mathrm{L}$, and ALT $>40 \mathrm{U} / \mathrm{L}$. Candidate predictors to enter the logistic multivariate regression were variables with $p<0.05$ in univariate analysis. Variables independently associated with the outcome at multivariate analysis together with those deemed as critically relevant in accordance with literature evidence and clinical experience were selected to fit the prediction model. The performance of the model was assessed by calculating the ROC curves and the corresponding area under the curve (AUC) values. The Hosmer-Lemeshow test was applied to assess the goodness-of-fit. All statistical analyses were carried out using International Business Machines (IBM) Statistical Package for the Social Sciences (SPSS) 21 (IBM Corporation: Armonk, NY, 10504).

\section{Role of the funding source}

The funders of the study did not have any role in data collection and in study design, data analysis, data interpretation, or writing of the report. The corresponding author had full access to all data in the study and had final responsibility for the decision to submit for publication.

\section{Results}

A total of 235 patients with COVID-19 were prospectively included in the cohort. Demographic and clinical characteristics of patients enrolled in the study are displayed in Table 1 . The majority of patients were male $(148,63 \%)$ and the mean age was 71 (SD 15.9). One hundred and ninety patients (81\%) suffered from at least one underlying illness, most frequently cardiovascular disease (47\%), neurological/psychiatric disorders (35\%), and diabetes (21\%).

The majority of patients had fever prior to admission (193, 82\%). Cough and dyspnoea were reported in 106 (45\%) and 87 (37\%) cases, respectively. The mean time from onset of symptoms and admission to hospital was 3.6 days (SD 3.2), while mean length of hospital stay was 13.2 days (SD 11.5). Patients received antiviral therapy according to national and regional recommendations (see Table 1). Steroids (methylprednisolone or dexamethasone), if prescribed, were administered for at least 5 days.

Table 1 Demographic and clinical characteristics of patients by COVID-19 disease progression ${ }^{\text {a }}$

\begin{tabular}{|c|c|c|c|c|c|}
\hline & \multirow[t]{2}{*}{ TOT $(n=235)$} & \multicolumn{2}{|c|}{ Disease progression $^{\mathrm{a}}$} & \multicolumn{2}{|l|}{ Univariate } \\
\hline & & No $(n=147)$ & Yes $(n=88)$ & OR $(95 \% \mathrm{CI})$ & $p$-value \\
\hline Age (years) > 65 & $157(67 \%)$ & $90(61 \%)$ & $67(76 \%)$ & $2.02(1.12$ to 3.65$)$ & 0.020 \\
\hline Gender (female) & $87(37 \%)$ & $58(40 \%)$ & $29(33 \%)$ & 0.75 (0.43 to 1.31$)$ & 0.318 \\
\hline Comorbidities (any) & $190(81 \%)$ & $119(81 \%)$ & $71(81 \%)$ & 0.98 (0.50 to 1.92$)$ & 0.959 \\
\hline Diabetes & 40/190 (21\%) & 27/119 (23\%) & $13 / 71(18 \%)$ & 0.76 (0.37 to 1.59$)$ & 0.475 \\
\hline Cancer & $33 / 190$ (17\%) & 22/119 (19\%) & $11 / 71(16 \%)$ & 0.81 (0.37 to 1.79$)$ & 0.599 \\
\hline Cardiovascular diseases & $90 / 190(47 \%)$ & 45/119 (38\%) & 45/71 (63\%) & 2.85 (1.55 to 5.23$)$ & 0.001 \\
\hline Renal diseases & $39 / 190$ (21\%) & 20/119 (17\%) & $19 / 71(27 \%)$ & 0.81 (0.89 to 3.69 ) & 0.103 \\
\hline Respiratory diseases & $34 / 190(18 \%)$ & $21 / 119(18 \%)$ & $13 / 71(18 \%)$ & 1.05 (0.49 to 2.25$)$ & 0.908 \\
\hline Liver diseases & 6/190 (3\%) & 4/119 (3\%) & 2/71 (3\%) & 0.83 (0.15 to 4.67$)$ & 0.836 \\
\hline $\mathrm{BMI}>30$ & $16 / 190(8 \%)$ & $12 / 119(10 \%)$ & 4/71 (6\%) & 0.53 (0.17 to 1.72$)$ & 0.292 \\
\hline Neurologic/Psychiatric diseases & $66 / 190(35 \%)$ & $38 / 119(32 \%)$ & 28/71 (39\%) & $1.39(0.75$ to 2.56$)$ & 0.294 \\
\hline \multicolumn{6}{|l|}{ Symptoms } \\
\hline Fever & $193(82 \%)$ & $120(82 \%)$ & $73(83 \%)$ & 1.09 (0.55 to 2.19$)$ & 0.798 \\
\hline Cough & $106(45 \%)$ & $68(46 \%)$ & $38(43 \%)$ & $0.88(0.52$ to 1.50$)$ & 0.646 \\
\hline Dyspnoea & $87(37 \%)$ & $41(28 \%)$ & $46(52 \%)$ & 2.83 (1.63 to 4.92$)$ & 0.000 \\
\hline \multicolumn{6}{|l|}{ Treatment } \\
\hline Steroids (at least 5 days) & $38 / 183(21 \%)$ & 26/124 (21\%) & $12 / 59(20 \%)$ & $0.96(0.45$ to 2.07$)$ & 0.922 \\
\hline Non-invasive ventilation & $26(11 \%)$ & $17(12 \%)$ & $9(10 \%)$ & 0.87 (0.37 to 2.05$)$ & 0.752 \\
\hline Invasive mechanical ventilation & $30(13 \%)$ & $2(1 \%)$ & $28(32 \%)$ & 33.83 (7.81 to 156.53$)$ & 0.000 \\
\hline \multicolumn{6}{|l|}{ Outcomes (days), mean (SD) } \\
\hline Time from symptoms onset to admission & $3.6(3.2)$ & $3.2(2.4)$ & $4.0(3.9)$ & $0.78(-0.08$ to 1.63$)$ & 0.076 \\
\hline Length hospital stay & $13.2(11.5)$ & $12.4(11.2)$ & $14.3(12)$ & $1.82(-1.24$ to 4.87$)$ & 0.243 \\
\hline
\end{tabular}

BMl: body mass index

${ }^{a}$ Disease progression is a composite outcome defined as: death and/or transfer to ICU within day- 11 after symptom onset and/or PaO $/ 2 / \mathrm{FiO}{ }_{2}$ ratio $<200$ on day 10 after symptom onset. Results are presented as mean (SD) and mean difference $(95 \% \mathrm{Cl})$ or frequencies $(\%)$ and OD $(95 \% \mathrm{Cl})$ as appropriate 
In $26(11 \%)$ cases non-invasive ventilation was required, while $30(13 \%)$ patients underwent invasive mechanical ventilation.

Eighty-eight (37\%) patients experienced poor outcome, e.g. $\mathrm{PaO}_{2} / \mathrm{FiO}_{2}$ ratio $<200$ (37 patients, $42 \%$ ), transfer to ICU (30 patients, 34\%), and death (47 patients, 53\%) by day-11 after symptoms onset. At univariate analysis (Table 1), patients with poor outcome were more likely to be older than 65 years (OR 2.02; 95\% CI 1.12-3.65 years, $p=0.020$ ), to suffer from cardiovascular disease (OR 2.85 ; $95 \%$ CI $1.55-5.23, p=0.001)$, and to have experienced dyspnoea during the 5 days prior to admission (OR 2.83; 95\% CI 1.63-4.92, $p<0.000)$. Blood test performed by day-5 of symptoms onset showed that mean values of CRP, NLR, LDH and CK were significantly higher compared with patients not experiencing progression of COVID-19 (Fig. 1). After applying the cut-offs set at the best point of sensitivity and specificity identified through the ROC curve, an association between disease progression and the above mentioned variables showing values above the cut-offs was observed (CRP: OR 7.38; 95\% CI 3.83-14.23, $p<0.000$; NLR: OR 3.43; 95\% CI 1.77-6.64, $p<0.000$; LDH: OR 6.70; 95\% CI 2.14-21.04, $p=0.001$; and CK: OR 3.83; 95\% CI 1.56-9.39, $p=0.003$ ) (Table 2).

Multivariate logistic regression showed that at day 5 presence of cardiovascular disease (OR 2.29; CI 95\% $1.12-4.68$; $p=0.023$ ), dyspnoea (OR 2.40; 95\% CI 1.19$4.84 ; p=0.015)$ and at least three abnormal laboratory findings among CRP $>80 \mathrm{U} / \mathrm{L}, \mathrm{ALT}>40 \mathrm{U} / \mathrm{L}, \mathrm{NLR}>4.5$, $\mathrm{LDH}>250 \mathrm{U} / \mathrm{L}$, and $\mathrm{CK}>80 \mathrm{U} / \mathrm{L}$ (OR 2.80; 95\% CI $1.35-5.81 ; p=0.006)$ were independently associated with COVID-19 progression (Table 3). A model combining the above mentioned variables with age $>65$ years old and male sex showed an AUC of 0.73 (95\% CI 0.66-0.81) for

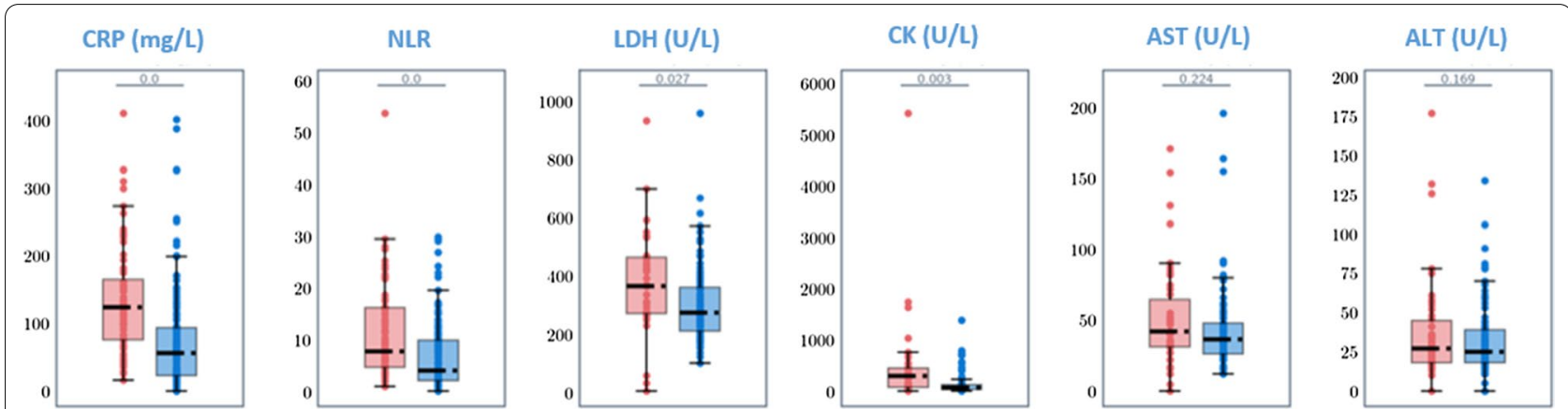

Fig. 1 Comparison of laboratory parameters at day-5 from symptoms onset between patents with (red) and without (blue) COVID-19 disease progression. Disease progression is a composite outcome defined as: death and/or transfer to ICU by day- 11 after symptom onset, and/or $\mathrm{PaO}_{2} / \mathrm{FiO} \mathrm{O}_{2}$ ratio < 200 on day 10 after symptom onset. CRP C-reactive protein, NLR neutrophils to lymphocytes ratio, LDH lactate dehydrogenase, CK creatine kinase, AST aspartate aminotransferase, ALT alanine transaminase. $p$-values are reported the top of each box plot

Table 2 Univariate analysis of laboratory parameters by COVID-19 progression ${ }^{\mathrm{a}}$

\begin{tabular}{|c|c|c|c|c|c|}
\hline & \multirow[t]{2}{*}{ TOT $(n=235)$} & \multicolumn{2}{|c|}{ Disease progression $^{\mathrm{a}}$} & \multicolumn{2}{|l|}{ Univariate } \\
\hline & & No $(n=147)$ & Yes $(n=88)$ & OR (95\% Cl) & $p$-value \\
\hline \multicolumn{6}{|l|}{ Laboratory parameters } \\
\hline $\mathrm{CRP}>80 \mathrm{mg} / \mathrm{L}$ & 87/196 (44\%) & $34 / 124$ (27\%) & $53 / 72(74 \%)$ & 7.38 (3.83 to 14.23 ) & 0.000 \\
\hline$N L R>4.5$ & 104/184 (57\%) & $54 / 117(46 \%)$ & $50 / 67(75 \%)$ & 3.43 (1.77 to 6.64$)$ & 0.000 \\
\hline $\mathrm{LDH}>250 \mathrm{U} / \mathrm{L}$ & 68/104 (65\%) & $37 / 69(54 \%)$ & $31 / 35(89 \%)$ & 6.70 (2.14 to 21.04$)$ & 0.001 \\
\hline $\mathrm{CK}>80 \mathrm{U} / \mathrm{L}$ & $56 / 102(55 \%)$ & 29/66 (44\%) & $27 / 36(75 \%)$ & $3.83(1.56$ to 9.39$)$ & 0.003 \\
\hline $\mathrm{ALT}>40 \mathrm{U} / \mathrm{L}$ & $35 / 148(24 \%)$ & 20/94 (21\%) & $15 / 54(28 \%)$ & 1.42 (0.66 to 3.09$)$ & 0.371 \\
\hline $\begin{array}{l}\text { At least } 3 \text { abnormal labora- } \\
\text { tory values }^{b}\end{array}$ & $66 / 210(31 \%)$ & $29(22 \%)$ & $37(49 \%)$ & $3.56(1.93$ to 6.56$)$ & 0.000 \\
\hline
\end{tabular}

CRP C-reactive protein, NLR neutrophils to lymphocytes ratio, $L D H$ lactate dehydrogenase, $C K$ creatine kinase, $A S T$ aspartate aminotransferase, $A L T$ alanine transaminase

${ }^{\text {a }}$ Disease progression is a composite outcome defined as: death and/or transfer to ICU within day-11 after symptom onset, and/or PaO $2 / \mathrm{FiO}_{2}$ ratio $<200$ on day 10 after symptom onset

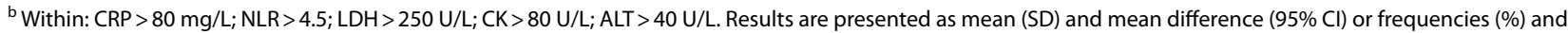
$\mathrm{OD}(95 \% \mathrm{Cl})$, as appropriate 
Table 3 Multivariate analysis of clinical characteristics and laboratory parameters by COVID-19 progression

\begin{tabular}{|c|c|c|}
\hline & OR $(95 \% \mathrm{Cl})$ & $p$-value \\
\hline Age (years) $>65$ & 1.53 (0.63 to 3.67 ) & 0.345 \\
\hline Cardiovascular diseases & 2.29 (1.12 to 4.68$)$ & 0.023 \\
\hline Dyspnoea & 2.40 (1.19 to 4.84$)$ & 0.015 \\
\hline $\begin{array}{l}\text { At least } 3 \text { abnormal laboratory } \\
\text { values }^{\text {a }}\end{array}$ & $2.80(1.35$ to 5.81$)$ & 0.006 \\
\hline Antibiotic therapy & 1.16 (0.57 to 2.36$)$ & 0.691 \\
\hline
\end{tabular}

a Within: CRP > 80 mg/L; NLR > 4.5; LDH > 250 U/L; CK > 80 U/L; ALT > 40 U/L

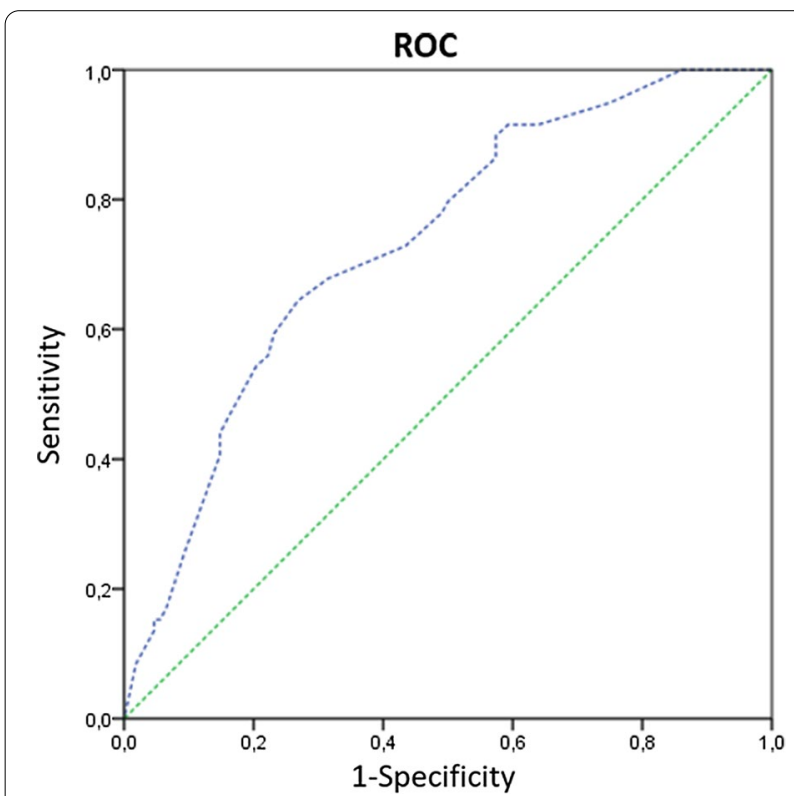

Fig. 2 COVID-19 disease progression by day-11 after onset of symptoms: Receiver operator characteristic (ROC) curve for discrimination, Area under the curve $(A \cup C)=0.733(95 \% \mathrm{Cl} 0.655-$ 0.810). Hosmer-Lemeshow Chi-squared $=8.061, p=0.427 ; \mathrm{DF}=8$ ). Variables included in the model: age $>65$ years, male sex, presence of cardiovascular disease, at least three abnormal parameters at day- 5 after symptom onset within: $\mathrm{CRP}>80 \mathrm{mg} / \mathrm{L} ; \mathrm{NLR}>4.5 ; \mathrm{LDH}>250 \mathrm{U} / \mathrm{L}$; $\mathrm{CK}>80 \mathrm{U} / \mathrm{L} ; \mathrm{ALT}>40 \mathrm{U} / \mathrm{L}$, and history of dyspnoea prior to admission

predicting disease progression. The Hosmer-Lemeshow goodness of fit test did not reach statistical significance $(p=0.427)$, indicating a good match of predicted risk over observed risk. The ROC curve for COVID-19 progression is displayed in Fig. 2.

\section{Discussion}

This study proposes an easy-to-use panel of five clinical and laboratory parameters to aid in the prediction of disease progression in COVID-19 patients. The model including age $>65$ years, male sex, cardiovascular disease, dyspnoea and at least three abnormal blood parameters among CRP $(>80 \mathrm{U} / \mathrm{L})$, ALT $(>40 \mathrm{U} / \mathrm{L}), \operatorname{NLR}(>4.5)$, $\mathrm{LDH}(>250 \mathrm{U} / \mathrm{L})$, and $\mathrm{CK}(>80 \mathrm{U} / \mathrm{L})$ shows fair discrimination ability (AUC 0.73 ). To our knowledge, this is the first study assessing predictors of disease progression at specific time-points starting from the onset of COVID19 symptoms.

Since the start of the pandemic, a wide range of predictive models and scores has been published with the common goal of informing clinical decision making and optimising resource allocation. An observational cohort of 1157 patients acutely admitted to two London hospitals analysed several demographics, clinical, laboratory and imaging factors likely to predict mortality, highlighting a correlation of male sex, older age, hypertension, chronic lung diseases and higher levels of lymphocytes, CRP and creatinine, among the others, with critical care admission and/or death [7]. A systematic review of ten prognostic models revealed that the most reported predictors of disease progression and mortality were age, sex, CRP, LDH and lymphocyte count [9]. Recently, the predictive value of NLR measured at hospital admission has been assessed in a prospective cohort, showing a high value in predicting disease deterioration, shock and death (all the areas under the curve $>0.80$ ) [13]. The vast majority of these models assessed the predictors by considering the hospital admission as baseline time-point [10-13], and share therefore the common drawback of including patients at varying stages of the disease.

In our cohort, more than one-third (37\%) of patients experienced disease progression by day- 11 of symptoms onset. Descriptive studies on clinical course of COVID19 revealed that the median time from onset of illness to acute respiratory distress syndrome and to ICU admission was 8-12 days and 9.5-12 days, respectively [14]. These findings would seem to indicate that clinical deterioration with the need of higher level of care may occur at a very early stage of the disease and suggest that setting "onset of symptoms" as baseline time-point leads to inclusion of patients with homogeneous disease length.

Older age and the presence of cardiovascular comorbidities were associated with a higher risk of unfavourable outcome, as highlighted by other studies [7, 15, 16]. Comorbidities, secondary bacterial infections, and altered cellular and humoral immune functions are more common in the elderly, thus increasing the risk of developing severe illness. A meta-analysis of 51 studies including a total of over 48,000 patients with confirmed COVID-19 infection showed that fatal outcomes with COVID-19 infection were strongly associated with diabetes, hypertension and cardiovascular diseases across all age groups, thus suggesting that the risk of poor outcome associated with cardiovascular diseases may be not 
affected by age [17]. In the present cohort, no statistically significant differences between male and female were observed with regards to the outcome, while in most of the studies the severity and mortality of COVID-19 have been reported to be higher in males than in females. Sex-related differences in immune system responses to pathogens has been observed, with female eliciting higher immune responses. Furthermore, RNA clearance has been reported to be delayed in male patients with COVID-19 [18].

Laboratory assessment on day- 5 of symptoms onset showed several parameters with significant mean differences between patients with or without poor outcome. In particular, poor outcome was associated with higher NLR, CRP, LDH, and CK, according with several studies [12, 19]. Lymphopenia and elevated neutrophil count suggest an alteration of lymphocyte function and are associated with elevated secretion of IL-6 and TNFalpha, since these inflammatory markers contribute to lymphocyte apoptosis as well as decreased proliferation of lymphocytes [20]. Several mechanisms are responsible of this alteration, including direct infection of the lymphocytes with SARS-CoV-2 virus, causing lymphocyte death or dysfunction. Furthermore, the pro-inflammatory state with increased cytokines is inversely correlated with the induction of granulopoiesis (resulting in neutrophilia) and suppressed lymphopoiesis in the bone marrow of patients with SARS-CoV infection [21].

This inflammatory state, when persistent and uncontrolled, may lead to acute respiratory distress syndrome and rapid deterioration of the clinical conditions. IL-6 upregulates hepatic CRP production, thus suggesting the plausibility of the clinical use of this inflammatory biomarker as a prognosis predictor for COVID-19 patients [9]. Similarly, LDH blood level increases in case of cell damage and is a marker of various inflammatory states. A systematic review and meta-analysis of 28 studies reporting LDH levels in severe vs. non-sever COVID19 patients confirmed that LDH level can be used as a COVID-19 severity marker and is a predictor of survival [22] CK, a marker of muscle damage, has been associated with a more severe COVID-19. Currently, it remains unclear whether increased levels of CK in COVID19 patients is due to a virus-triggered inflammatory response or direct muscle toxicity [23]. Elevation of liver transaminases during SARS-CoV-2 infection has been frequently reported. Possible pathophysiology mechanisms include direct effect of the virus, liver injury mediated by uncontrolled immune response drug toxicity and ischemic hepatitis due to multiorgan dysfunction [24]. Based on these evidences and on our findings, patients affected by COVID-19 may benefit from blood testing including inflammation markers, complete blood count and transaminases within the 1st week after symptoms onset to evaluate the risk of developing a life-threatening disease.

In a recent work, a large group of Italian experts was invited to complete an online survey through the PAPRIKA (Potentially All Pairwise RanKings of all possible Alternatives) method [25] with the aim of determining the weights of several criteria for prioritizing COVID-19 patients for hospitalization [26]. Among a list of criteria, including age, body mass index, comorbidities, findings at chest X-ray, CRP, and duration of symptoms among others, the highest weights were attributed to $\mathrm{PaO}_{2}$ and peripheral oxygen saturation, denoting the well-known central role of respiratory findings in the assessment of the risk of rapid deterioration of COVID19 patients. These findings, alongside those outlined in our study, suggest that in addition to considering symptoms onset and duration, the typology of symptoms should also be taken into account in an early risk assessment of COVID-19 deterioration.

This study has several limitations. First, the sample size limits the accuracy of the findings. Second, the study was performed in a single centre, hampering the generalizability and the applicability of the results to other settings. Third, the model did not undergo external validation and might be at risk of overfitting. However, we tried to mitigate overfitting by decreasing the number of predictors. The results from this study highlight the importance of relying on homogeneous populations with same length of disease in order to build the best possible prediction model for disease progression in COVID-19 patients and allow optimal treatment and resource allocation.

This study shows how an easy-to-use panel of five laboratory and clinical parameters applied in patients with COVID-19 at day-5 of symptoms onset can predict disease progression with fair discriminatory power. It further suggests that the onset of symptoms might represent a useful and reliable baseline time-point for developing prognostic models. The assessment of few variables at the right time may contribute to early identification of patients at major risk of developing life-threatening COVID-19. The model can also play a role as a tool to increase homogeneity of population in clinical trials on COVID-19 treatment in hospitalized patients. A validation study is needed to evaluate whether this model can reliably inform decision making and identify proper level-of-care requirements for hospitalized patients.

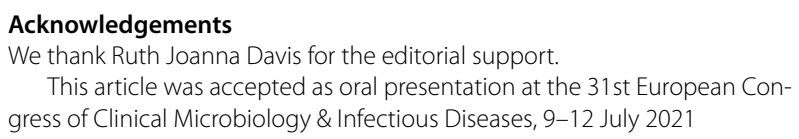




\section{Authors' contributions}

EG, AV, MC, GB, ER, NS, DM and SM collected the data. EG performed the statistical analysis and wrote the first draft of the manuscript. ET, EG, DG, CM and ER contributed in the conceptualisation of the study. PDN reviewed the literature, edited and revised all the draft of the manuscript. AG did the visualization. AS and $M C$ reviewed the manuscript. ET supervised the work, provided advice to the statistical analysis, and reviewed all the drafts of the manuscript. All authors read and approved the final manuscript.

\section{Funding}

This study is part of React-COVID-19 project funded by Fondazione CARIVERONA.

\section{Availability of data and materials}

The datasets analysed during the current study are available from the corresponding author on reasonable request.

\section{Declarations}

\section{Ethics approval and consent to participate}

The study was approved by the hospital Institutional Review Board ("Comitato etico per la sperimentazione clinica delle province di Verona e Rovigo", 2577CESC). A written informed consent for study participation was obtained from the patients or from the legal guardian on enrolment. The study protocol is performed in accordance with the relevant guidelines. Further information are available at ClinicalTrials.gov NCT04497194.

\section{Consent for publication}

All the authors gave their consent for the publication.

\section{Competing interests}

The authors declare no competing interests.

\section{Author details}

1 Division of Infectious Diseases, Department of Diagnostics and Public Health, University of Verona, P.le L.A. Scuro 10, 37134 Verona, Italy. ${ }^{2}$ Department of Medicine, Section of Internal Medicine, University of Verona, EuroBloodNet Referral Center for Iron Metabolism Disorders, Azienda Ospedaliera Universitaria Integrata Verona, 37138 Verona, Italy. ${ }^{3}$ Cardio-Thoracic Department, Respiratory Unit, Azienda Ospedaliera Universitaria Integrata Verona, 37124 Verona, Italy. ${ }^{4}$ Department of Radiology, University of Verona, Piazzale L.A. Scuro, 10, 37100 Verona, Italy.

Received: 28 April 2021 Accepted: 20 August 2021

Published online: 28 August 2021

\section{References}

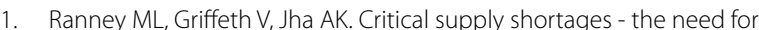
ventilators and personal protective equipment during the Covid-19 pandemic. N Engl J Med. 2020;382(18):e41. https://doi.org/10.1056/NEJMp 2006141

2. World Health Organization. Coronavirus Disease (COVID-19) dashboard. https://covid19.who.int/table. Accessed 27 July 2021.

3. Wu Z, McGoogan JM. Characteristics of and Important lessons from the Coronavirus Disease 2019 (COVID-19) outbreak in China: summary of a report of 72314 cases from the Chinese Center for Disease Control and Prevention. JAMA. 2020;323(13):1239-42. https://doi.org/10.1001/jama. 2020.2648.

4. Richardson S, Hirsch JS, Narasimhan M, et al. Presenting characteristics, comorbidities, and outcomes among 5700 patients hospitalized with COVID-19 in the New York City Area. JAMA. 2020;323:2052.

5. European Centre for Disease Prevention and Control. COVID-19 surveillance report. https://covid19-surveillance-report.ecdc.europa.eu/. Accessed 20 Jan 2021

6. Cohen PA, Hall LE, John JN, Rapoport AB. The early natural history of SARS-CoV-2 infection: clinical observations from an urban, ambulatory COVID-19 clinic. Mayo Clin Proc. 2020;95(6):1124-6. https://doi.org/10. 1016/j.mayocp.2020.04.010.
7. Galloway JB, Norton S, Barker RD, et al. A clinical risk score to identify patients with COVID-19 at high risk of critical care admission or death: an observational cohort study. J Infect. 2020. https://doi.org/10.1016/j.jinf. 2020.05.064 (published online ahead of print, 2020 May 29).

8. Huang C, Wang Y, Li X, Ren L, Zhao J, Hu Yet al. Clinical features of patients infected with 2019 novel coronavirus in Wuhan, China. Lancet. 2020;395(10223):497-506. https://doi.org/10.1016/S0140-6736(20)301835. Erratum in: Lancet. 2020 Jan 30. (Epub 2020 Jan 24).

9. Wynants L, Van Calster B, Collins GS, et al. Prediction models for diagnosis and prognosis of covid-19 infection: systematic review and critical appraisal [published correction appears in BMJ. 2020 Jun 3;369:m2204]. BMJ. 2020;369:m1328. https://doi.org/10.1136/bmj.m1328. (Published 2020 Apr 7)

10. Shang Y, Liu T, Wei Y, Li J, Shao L, Liu M, et al. Scoring systems for predicting mortality for severe patients with COVID-19. EClinicalMedicine. 2020;3(24):100426. https://doi.org/10.1016/j.eclinm.2020.100426.

11. Wendel Garcia PD, Fumeaux T, Guerci P, Heuberger DM, Montomoli J, Roche-Campo F, et al. Prognostic factors associated with mortality risk and disease progression in 639 critically ill patients with COVID-19 in Europe: initial report of the international RISC-19-ICU prospective observational cohort. EClinicalMedicine. 2020;25:100449. https://doi.org/10. 1016/j.eclinm.2020.100449.

12. Mesas AE, Cavero-Redondo I, Álvarez-Bueno C, Sarriá Cabrera MA, MaffeideAndrade S, Sequí-Dominguez I, Martínez-Vizcaíno V. Predictors of in-hospital COVID-19 mortality: a comprehensive systematic review and meta-analysis exploring differences by age, sex and health conditions. PLOS ONE. 2020;15(11):e0241742. https://doi.org/10.1371/journal.pone. 0241742.

13. Zeng ZY, Feng SD, Chen GP, Wu JN. Predictive value of the neutrophil to lymphocyte ratio for disease deterioration and serious adverse outcomes in patients with COVID-19: a prospective cohort study. BMC Infect Dis. 2021;21(1):80. https://doi.org/10.1186/s12879-021-05796-3.

14. Centers for Disease Control and Prevention. Interim clinical guidance for management of patients with confirmed Coronavirus Disease (COVID19). https://www.cdc.gov/coronavirus/2019-ncov/hcp/clinical-guidancemanagement-patients.html\#: :text=Among\%20patients\%20in\%20mul tiple\%20early,median\%20time\%20from\%20onset\%20of. Accessed 20 Jan 2021.

15. Dorjee K, Kim H, Bonomo E, Dolma R. Prevalence and predictors of death and severe disease in patients hospitalized due to COVID-19: a comprehensive systematic review and meta-analysis of 77 studies and 38,000 patients. PLoS ONE. 2020;15(12):e0243191. https://doi.org/10.1371/journ al.pone.0243191.

16. Li L, Sun W, Han M, Ying Y, Wang Q. A study on the predictors of disease severity of COVID-19. Med Sci Monit. 2020;26:e927167. https://doi.org/10. 12659/MSM.927167 (Published 2020 Sep 23).

17. Bae S, Kim SR, Kim M-N. Impact of cardiovascular disease and risk factors on fatal outcomes in patients with COVID-19 according to age: a systematic review and meta-analysis. Heart. 2021;107:373-80. https://doi.org/10. 1136/heartjnl-2020-317901

18. Xu K, Chen Y, Yuan J, Yi P, Ding C, Wu W, et al. Factors associated with prolonged viral RNA shedding in patients with coronavirus disease 2019 (covid-19). Clin Infect Dis. 2020;71:799-806.

19. Izcovich A, Ragusa MA, Tortosa F, et al. Prognostic factors for severity and mortality in patients infected with COVID-19: a systematic review. PLoS ONE. 2020;15(11):e0241955. https://doi.org/10.1371/journal.pone.02419 55.

20. Fan BE, Chong VCL, Chan SSW, et al. Hematologic parameters in patients with COVID-19 infection. Am J Hematol. 2020;95(6):E131-4. https://doi. org/10.1002/ajh.25774.

21. Tavakolpour S, Rakhshandehroo T, Wei EX, Rashidian M. Lymphopenia during the COVID-19 infection: What it shows and what can be learned. Immunol Lett. 2020;225:31-2.

22. Szarpak L, Ruetzler K, Safiejko K, et al. Lactate dehydrogenase level as a COVID-19 severity marker. Am J Emerg Med. 2020. https://doi.org/10. 1016/j.ajem.2020.11.025 (published online ahead of print, 2020 Nov 15).

23. Pitscheider L, Karolyi M, Burkert FR, Helbok R, Wanschitz JV, Horlings C, Pawelka E, Omid S, Traugott M, Seitz T, Zoufaly A, Lindeck-Pozza E, Wöll E, Beer R, Seiwald S, Bellmann-Weiler R, Hegen H, Löscher WN. Muscle 
involvement in SARS-CoV-2 infection. Eur J Neurol. 2021. https://doi.org/ 10.1111/ene.14564.

24. Kumar-M P, Mishra S, Jha DK, et al. Coronavirus disease (COVID-19) and the liver: a comprehensive systematic review and meta-analysis. Hepatol Int. 2020;14(5):711-22. https://doi.org/10.1007/s12072-020-10071-9.

25. Hansen $P$, Ombler F. A new method for scoring additive multi-attribute value models using pairwise rankings of alternatives. J Multi-Criteria Decis Anal. 2008;15:87-107.

26. De Nardo P, Gentilotti E, Mazzaferri F, Cremonini E, Hansen P, Goossens $\mathrm{H}$, et al. Multi-criteria decision analysis to prioritize hospital admission of patients affected by COVID-19 in low-resource settings with hospital-bed shortage. Int J Infect Dis. 2020;98:494-500.

\section{Publisher's Note}

Springer Nature remains neutral with regard to jurisdictional claims in published maps and institutional affiliations.
Ready to submit your research? Choose BMC and benefit from:

- fast, convenient online submission

- thorough peer review by experienced researchers in your field

- rapid publication on acceptance

- support for research data, including large and complex data types

- gold Open Access which fosters wider collaboration and increased citations

- maximum visibility for your research: over $100 \mathrm{M}$ website views per year

At BMC, research is always in progress.

Learn more biomedcentral.com/submissions 